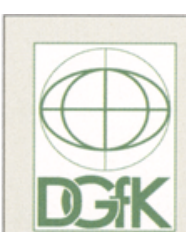

KN Kartographische Nachrichten Journal of Cartography and Geographic Information 66. Jahrgang, April 2016, Heft 2

Herausgeber: Deutsche Gesellschaft für Kartographie e.V. (DGfK) - Gesellschaft für Kartographie und Geomatik, vertreten durch den Präsidenten Prof. Dr. Manfred Weisensee

Organ der Deutschen Gesellschaft für Kartographie e.V. (DGfK), der Schweizerischen Gesellschaft für Kartografie (SGK) und der Österreichischen Kartographischen Kommission (ÖKK) in der Österreichischen Gesellschaft für Geographie (0̄GG).

Schriftleitung

Hauptschriftleiter

Prof. Dr. rer. nat. habil. Mark Vetter (zuståndig für Aufsätze, Berichtel. Hochschule Karlsruhe - Technik und Wirtschaft,

Moltkestr. 30, 76133 Karistuhe: redaktionekartographische-

nachrichten.de, Fon: 0049(0) 7219252599

Weitere Schriftleiter

Prof. Dr. phil. Frank Dickmann (Zweiter Schrifttleiter, zustăndig für Nachrichten aus Hochschulen und Institutionen, Rezensionen. Ruhr-Universităt Bochum, Geographisches Institut, Postfach, 44780 Bochum; frank dickmann@ @ubde

Fon: 0049(0) 234 32-23379; Fax: 0049(0) 234 32-14964

Dipl.-Ing. Andreas Gollenstede (zuständig für Geoinformation aktuell) Haareneschstr. 91, 26121 Oldenburg; kn@gollenstede.com Fon: 0049(0) $4417779-545$; Fax: 0049(0) 441 7779-750 Dr.-Ing. Thomas Chudy (zustāndig für DGfK-, SKG- und ÖGG-

Nachrichten). Martin-Luther-Universität Halle-Wittenberg, Institut für Agrar- und Ernährungswissenschaften, Karl-Freiherr-vonFritsch-StraBe 4, 06120 Halle/Saale, Fon: 0049(0) 3455522448 ; Fax: 0049(0) 3455527109

Prof. Dr. Reinhard Zölitz (zustăndig für Nachrichten aus Hochschulen und Institutionen), Institut für Geographie und Geologie, Kartographie und GIS, Friedrich-Ludwig-Jahn-Str. 16

D-17489 Greifswald, Fon 03834864523, Fax:03834864501.

E-Mail: zoelitz@uni-greifswald.de

Mitarbeit für die Schweiz: Prof. Dr. Lorenz Hurni. Institut für Kartografie, ETH Hönggerberg, 8093 Zürich; hurni@karto.baug.ethz. ch. Fon: 0041 16333033; Fax: 0041 1631153

Mitarbeit für Österreich: Univ.-Prof. Dr. Wolfgang Kainz. Institut für Geographie und Regionalforschung, Universităt Wien, Universitätsstraße 7/5, 1010 Wien; wolfgang.kainz@univie.ac.at Fon: 0043 14277-8640; Fax: 0043 14277-9531

Ehrenschriftleiter

Prof. i. R. Dr. Jürgen Dodt, Witten

Dr. rer. nat. h. c. Rolf Harbeck, Bonn

Editorial Board

Prof. Dr. H. Asche, Potsdam; Prof. Dr. Manfred Buchroithner, Dresden; Prof. Dr. Dirk Burghardt, Dresden; Prof. i. R. Dr. Jürgen Dodt, Witten; Prof. Dr. D. Dransch, Potsdam; Prof. Dr. M. Ehlers Osnabrück, Berlin; Prof. Dr. S. I. Fabrikant, Zürich, Schweiz; Ass-Prof. Dr. S. Fuhrmann, Fairfax, USA; Prof. Dr. G. Gartner, Wien, Ass-Prof. Dr. S. Fuhrmann, Fairfax, USA; Prof. Dr. G. Gartner, Wiec,
Österreich; Prof. Dr. D. Grünreich, Ronnenberg; Dr. Rolf Harbeck, Bonn; Prof. Dr. A. Hüttermann, Ludwigsburg; Prof. Dr. L. Hurni, Zürich; Prof. Dr. W. Kainz, Wien; Prof. Dr. P. Kammerer, München; Prof. Dr. W. G. Koch, Dresden; Prof. Dr. Menno-Jan Kraak, Twente, Netherlands; Prof. Dr. Jukka Krisp, Augsburg; Prof. Dr.-Ing. L Meng, München: Prof. Dr. J. Schiewe, Hamburg; PD Dr.-Ing. J. Schoppmeyer, Bonn; Prof. Dr.J.Schweikart, Berlin; Prof. Dr.-Ing. M. Sester, Hannover; Prof. Dr. J. Siemer, Regina, Kanada; Prof. Dr. R. Zölitz, Greifswald.

Manuskripte richten Sie bitte an den zustāndigen Schriftleiter Hinweise zur Gestaltung des Manuskripts finden Sie unter wwwkkartographische-nachrichten de > Autorenhinweise.

Reviewverfahren

Wissenschaftliche Beitrăge werden im Rahmen eines Peer-ReviewVerfahrens begutachtet. Trotz sorgfältiger Redigierung übernehmen Herausgeber, Redaktion und Verlag keine Haftung für die inhaltliche Richtigkeit der Veröffentlichungen.

Die KN Kartographische Nachrichten ist in der internationalen

Zitationsdatenbank Scopus gelistet.

Die DGfK ist Mitglied der

Internationalen Kartographischen

Vereinigung IKV/ICA.

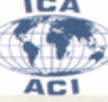

Verlag: Kirschbaum Verlag GmbH, Siegfriedstraße 28 ,

D-53179 Bonn / Postfach 210209, D-53157 Bonn.

Fon: (0228) 95453-0, Fax: (0228) 95453-27;

Internet: $h$ ttp://www.kirschbaum.de, E-Mail: info@kirschbaum.de

Anzeigenleiter: Volker Rutkowski; zurzeit ist Anzeigenpreisliste

Nr. 51 vom 1.10.2015 gültig

Bezugspreise und ISSN: Inland/Ausland Jahresabonnement

inkl. E-Paper und elektronischem Archiv 74,- Euro zzgl.

Versand 9,80,- Euro (jeweils einschl. MwSt).

Einzelheft 18- Euro zzgl. Versand

Die Zeitschrift erscheint alle zwei Monate. ISSN 0022-9164.

Kündigungsfrist 6 Wochen zum Auslaufen des Abonnement-

Zeitraumes.

Satz: EMS Eckert Medienservice, 53359 Rheinbach

Druck: johnen-druck GmbH \& Co. KG, Industriegebiet Bornwiese,

54470 Bernkastel-Kues

\title{
Ein neues Kleid für die KN
}

\section{Liebe Leserin, lieber Leser,}

Kenner der Verlagsbranche sagen, alle paar Jahre brauche eine Zeitschrift ein neues Layout. Nur wer sich ständig neu erfindet, bleibt interessant und im Gespräch. Gleichzeitig steigt ständig der Kostendruck. In diesem Lichte heißt es auch bei der KN: „Es tut sich was.“

Das neue Kleid der KN haben verschiedene Expertinnen und Experten der Gestaltungswelt

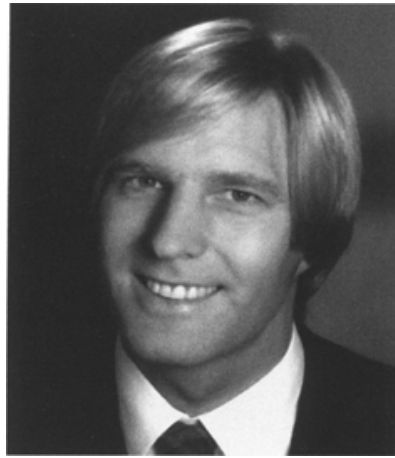

Mark Vetter in Kooperation mit dem Vorstand der DGfK und dem Verlag erschaffen. Ein besonderer Dank gilt Herrn Andreas Lörzer vom Kirschbaum Verlag, der diese Vorschläge gestalterisch umgesetzt hat.

Was ist neu? Das Entfallen der Begrenzungslinien lockert den Text optisch auf. Gemeinsam mit der von Otl Aicher 1988 entwickelten Schrifttype Rotis wurde eine klarere Formensprache im Textteil erreicht. Dies fördert die Vermittlung von Inhalten. Und spart Platz, zugunsten einer besseren Ressourcennutzung. Ein persönliches Anliegen war mir die Neugestaltung des Covers. Großformatige Darstellungen finden künftig auf der neu gestalteten Titelseite der KN mehr Raum. So kommen die wunderbaren Karten und Abbildungen unserer Wissenschaft noch besser zur Geltung. Und unterstreichen den Charakter der KN als Zeitschrift für Geovisualisierung. Eine weitere Neuerung: der Postumschlag fällt in Zukunft weg. So wird die KN künftig noch kosteneffizienter.

In dieser Ausgabe dürfen Sie sich auf vier Fachbeiträge freuen: Dennis Edler und Frank Dickmann gehen in ihrer Betrachtung darauf ein, wie die Darstellung von Räumen in Karten von Videospielen in der Vergangenheit gelöst wurde. Florian Hruby diskutiert in seinem Beitrag die Vor- und Nachteile von Choroplethenkarten. Detlef Günther-Diringer zeigt den aktuellen technischen Stand der 3D-Visualisierung von urbanen Strukturen am beeindruckenden 3D-Stadtmodell von Karlsruhe. Schließlich gibt Peter Ladstätter - als Vorgriff auf seinen KeynoteVortrag für die geoinfo.potsdam.2016/64. Deutscher Kartographentag seine Vision von zukünftigen Geschäftsmodellen für Karten und Geoinformationen kund.

Ich wünsche Ihnen viel Freude beim Lesen der KN 2/16.

Ihr

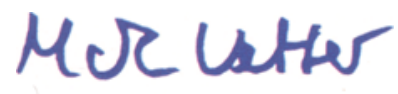

